Research Article

\title{
Measurement of the Euler Angles of Wurtzitic ZnO by Raman Spectroscopy
}

\author{
Wu Liu, ${ }^{1,2}$ Qiu Li, ${ }^{1}$ Gang Jin, ${ }^{1}$ and Wei Qiu ${ }^{3}$ \\ ${ }^{1}$ Tianjin Key Laboratory of High Speed Cutting and Precision Machining, School of Mechanical Engineering, \\ Tianjin University of Technology and Education, Tianjin 300222, China \\ ${ }^{2}$ School of Mechanical and Automotive Engineering, Zhejiang University of Water Resources and Electric Power, \\ Hangzhou 310018, China \\ ${ }^{3}$ Tianjin Key Laboratory of Modern Engineering Mechanics, School of Mechanical Engineering, Tianjin University, \\ Tianjin 300072, China
}

Correspondence should be addressed to Qiu Li; qiuli_tj@163.com and Wei Qiu; daniell_q@hotmail.com

Received 29 January 2017; Accepted 23 April 2017; Published 8 August 2017

Academic Editor: Jau-Wern Chiou

Copyright () $2017 \mathrm{Wu}$ Liu et al. This is an open access article distributed under the Creative Commons Attribution License, which permits unrestricted use, distribution, and reproduction in any medium, provided the original work is properly cited.

\begin{abstract}
A Raman spectroscopy-based step-by-step measuring method of Euler angles $(\varphi, \theta$, and $\psi)$ was presented for the wurtzitic crystal orientation on a microscopic scale. Based on the polarization selection rule and coordinate transformation theory, a series of analytic expressions for the Euler angle measurement using Raman spectroscopy were derived. Specific experimental measurement processes were presented, and the measurement of Raman tensor elements and Euler angles of the $\mathrm{ZnO}$ crystal were implemented. It is deduced that there is a trigonometric functional relationship between the intensity of each Raman bands of wurtzite crystal and Euler angle $\psi$, the polarization direction of incident light under different polarization configurations, which can be used to measure the Euler angles. The experimental results show that the proposed method can realize the measurement of Euler angles for wurtzite crystal effectively.
\end{abstract}

\section{Introduction}

The wide-band gap $\mathrm{ZnO}$ semiconductor material with hexagonal wurtzite crystal structure has excellent photoelectric properties. It is expected to become the next generation of the UV photoelectric material with excellent performance. And also, it has found wide extensive application prospects in many areas, such as piezoelectric gas sensor and pressure-sensitive sensor [1-3]. Since crystals often have different performances (e.g., electrical conductivity, thermal expansion coefficient, and mechanical strength) in different crystallographic orientations, fully grasping the crystallographic orientation parameters is necessary in the structural design of $\mathrm{ZnO}$ devices and mechanical behavior prediction for material microstructure. Namely, the relationship between crystallographic orientation and the observable characteristic direction of the device should be grasped. In short, the geometric relationship between the crystal coordinate system and specimen coordinate system, that is, Euler angles, needs to be determined.

The existing measurement methods of crystallographic orientations included etch-pit technique [4], neutron diffraction [5], electron backscatter diffraction (EBSD) [6-8], X-ray diffraction $[9,10]$, and micro-Raman spectroscopy [11]. For the etch-pit technique, it is destructive and restricted by a special erodent. The neutron diffraction method needs a long measurement time, and the neutron source construction and operation are expensive. The X-ray diffraction pattern may show no peaks at all in the whole scanning range when the deviation angle between the crystal plane and the sample surface is relatively large, making it difficult to assess the actual crystal orientation in such a case. The determination by micro-Raman spectroscopy has several advantages: (1) it needs no special preparation for the sample and is nondestructive, (2) the sample can be exposed to the air during the measurement, (3) spatial resolution is high $(\sim 1 \mu \mathrm{m})$, 


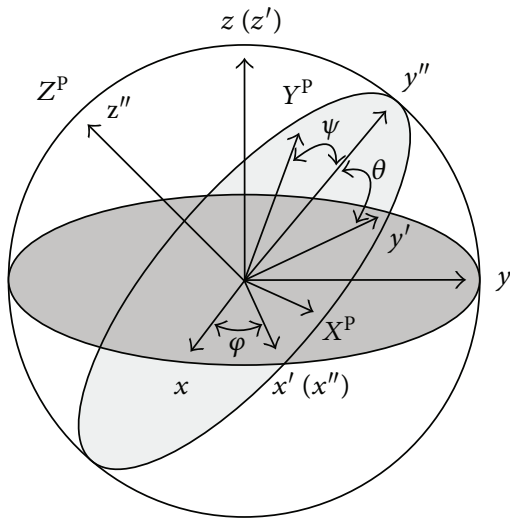

(a)

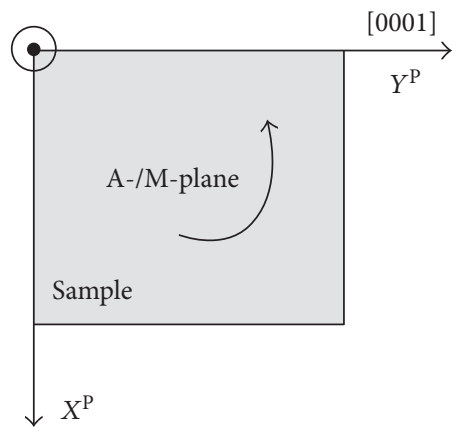

(c)

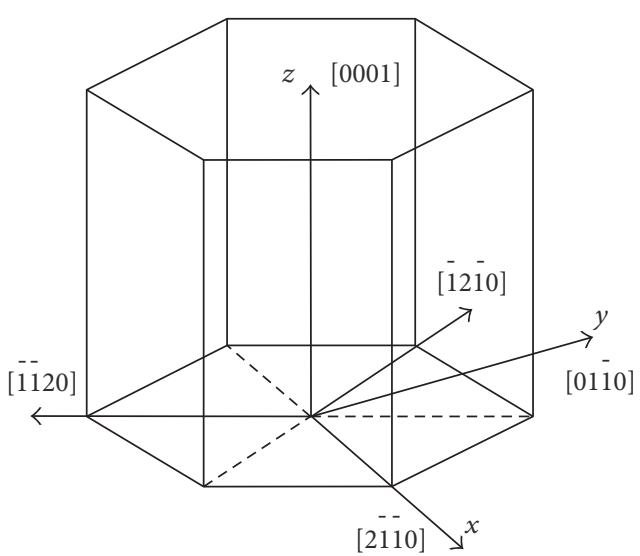

(b)

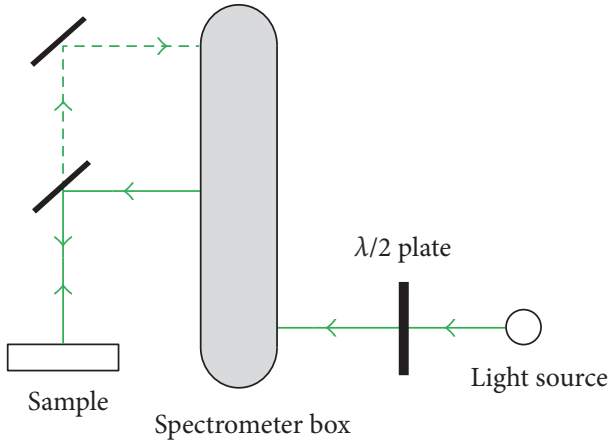

(d)

FIGURE 1: (a) The relationship between specimen coordinate system and crystal coordinate system; (b) the relationship between the hexagonal coordinates and Cartesian coordinates for wurtzitic crystal; (c) A-/M-plane $\mathrm{ZnO}$ sample initial position; (d) optical path in the Euler angle measurement experiment.

and (4) time required for the determination is relatively short. Hopkins and Farrow have determined the local crystallographic orientations of laser-recrystallized polycrystalline silicon in silicon-on-insulator (SOI) structures using polarized Raman spectroscopy [12]. Munisso et al. also employed this method to determine the crystallographic orientation of polycrystalline alumina [13]. The Euler angles of multicrystalline silicon have been determined by Becker et al. with Raman spectroscopy during the stress measurement, though this method involves complicated formulas and tedious calculations [14]. The validity of the Raman polarization analysis for the crystallographic orientation determination of the diamond and sapphire crystal has been demonstrated. However, the Raman measurement theory and method for the Euler angles of wurtzite crystal are still in the study stage.

In this article, based on the polarization properties of Raman spectra, the step-by-step measuring method for the Euler angle of the wurtzitic crystal is proposed. First, the theoretical formulas for the Euler angle measurement by Raman spectroscopy were deduced for wurtzite crystal. Subsequently, the specific experimental processes were given for the measurement of the Euler angles of a $\mathrm{ZnO}$ crystal. Using the sample with a known crystal orientation (A-plane), the Raman tensor elements of $\mathrm{ZnO}$ crystal were determined for the first time. Based on the proposed method, the Euler angles of the $\mathrm{M}$-plane $\mathrm{ZnO}$ were measured step by step. Then, the experimental results were compared with the theoretical value to verify the validity of the presented method.

\section{Theory}

Crystal coordinate system is represented by $x y z$. The relationship between the hexagonal coordinates and Cartesian coordinates of wurtzite crystal is shown in Figure 1(a), where $x\|[2 \overline{1} \overline{1} 0], y\|[01 \overline{1} 0]$, and $z \|[0001]$.

Specimen coordinate system is represented by $X^{\mathrm{P}} Y^{\mathrm{P}} Z^{\mathrm{P}}$. The relationship between specimen coordinates and crystal coordinates is shown in Figure 1(b). $\varphi, \theta$, and $\psi$ are the Euler angles which transform the crystal coordinate system to the specimen coordinate system. The values of $\varphi, \theta$, and $\psi$ are between 0 and $2 \pi$.

The theoretical value of the Euler angles of the crystallographic orientation known sample can be calculated according to the relationship between the Miller indices and the Euler angles. The relationship between the Miller index of the hexagonal system, $(h k i l)[u v t w]$, and the Euler angles can be expressed as follows [15]: 


$$
\begin{aligned}
& {\left[\begin{array}{cc}
\frac{3 u}{2 d_{u v w}} & \frac{\sqrt{3} h}{d_{h k l}} \\
\frac{\sqrt{3}(u+2 v)}{2 d_{u v w}} & \frac{h+2 k}{d_{h k l}} \\
\frac{w \cdot c}{a \cdot d_{u v w}} & \frac{\sqrt{3} l \cdot a}{c \cdot d_{h k l}}
\end{array}\right]} \\
& =\left[\begin{array}{cc}
\cos \varphi \cdot \cos \psi-\cos \theta \cdot \sin \varphi \cdot \sin \psi & \sin \theta \cdot \sin \psi \\
-\cos \varphi \cdot \cos \psi-\sin \varphi \cdot \cos \theta \cdot \cos \psi & \sin \theta \cdot \cos \psi \\
\sin \varphi \cdot \sin \theta & \cos \theta
\end{array}\right],
\end{aligned}
$$

where (hkil) represent the crystal orientation index at the $X^{\mathrm{P}}$ direction and $[u v t w]$ represent the crystal plane index of the measuring plane; $a$ and $c$ are lattice constants. For $\mathrm{ZnO}, d_{u v w}=\left[9 u^{2} / 4+3(u+2 v)^{2} / 4+w^{2}(c / a)^{2}\right]^{1 / 2}, d_{h k l}=$ $\left[3 h^{2}+3(h+2 k)^{2}+3 l^{2}(a / c)^{2}\right]^{1 / 2}$, and $c / a=1.602$.

In actual Raman scattering experiments, the geometric configuration of the experiment can be defined according to the Porto formalism [16] as follows: $\mathbf{k}_{i}\left(\mathbf{e}_{i}, \mathbf{e}_{s}\right) \mathbf{k}_{s}=$ propagation direction of incident light (polarization direction of incident light, polarization direction of scattering light) and propagation direction of scattering light. Mathematically, the Raman scattering intensity $I$ depends on the polarization vectors of both the incident $\left(\mathbf{e}_{i}\right)$ and scattered $\left(\mathbf{e}_{s}\right)$ lights:

$$
I=C\left|\mathbf{e}_{i} \cdot \mathbf{R}_{j} \cdot \mathbf{e}_{s}\right|^{2},
$$

where $C$ is a constant and $\mathbf{R}_{j}$ is the Raman tensor of the phonon $j$ in crystal coordinate system. Loudon derived the Raman tensors for each of the 32 crystal classes (symmetry point groups). As for wurtzite crystal, there are five Raman tensors that can be expressed in the crystal coordinate system $x\|[2 \overline{1} \overline{1} 0], y\|[01 \overline{1} 0]$, and $z \|[0001]$ as follows [17]:

$$
\begin{aligned}
\mathbf{R}_{A_{1}(z)} & =\left(\begin{array}{ccc}
a^{\prime} & 0 & 0 \\
0 & a^{\prime} & 0 \\
0 & 0 & b^{\prime}
\end{array}\right), \\
\mathbf{R}_{E_{1}(x)} & =\left(\begin{array}{ccc}
0 & 0 & -c^{\prime} \\
0 & 0 & 0 \\
-c^{\prime} & 0 & 0
\end{array}\right), \\
\mathbf{R}_{E_{1}(y)} & =\left(\begin{array}{ccc}
0 & 0 & 0 \\
0 & 0 & c^{\prime} \\
0 & c^{\prime} & 0
\end{array}\right), \\
\mathbf{R}_{E_{2}(x)} & =\left(\begin{array}{ccc}
0 & d^{\prime} & 0 \\
d^{\prime} & 0 & 0 \\
0 & 0 & 0
\end{array}\right), \\
\mathbf{R}_{E_{2}(y)} & =\left(\begin{array}{ccc}
d^{\prime} & 0 & 0 \\
0 & -d^{\prime} & 0 \\
0 & 0 & 0
\end{array}\right),
\end{aligned}
$$

where $a^{\prime}, b^{\prime}, c^{\prime}$, and $d^{\prime}$ are Raman tensor constants and the superscripts $A_{1}(z), E_{1}(x), E_{1}(y), E_{2}(x)$, and $E_{2}(y)$ denote Raman vibration modes of wurtzite crystal.

Given that the matrix of Euler angles $\Phi_{x y z}$ (and its inverse matrix $\bar{\Phi}_{x y z}$ ) transforms the Raman tensor in crystal coordinate system into that in specimen coordinate system, the following expression is given:

$$
\mathbf{R}_{j}^{\prime}=\bar{\Phi}_{x y z} \mathbf{R}_{j} \Phi_{x y z},
$$

where

$$
\begin{aligned}
\Phi_{x y z} & =\left|\begin{array}{ccc}
\cos \varphi \cdot \cos \psi-\cos \theta \cdot \sin \varphi \cdot \sin \psi & -\cos \varphi \cdot \sin \psi-\sin \varphi \cdot \cos \varphi \cdot \cos \psi & \sin \varphi \cdot \sin \theta \\
\cos \varphi \cdot \cos \theta \cdot \sin \psi+\cos \psi \cdot \sin \varphi & \cos \varphi \cdot \cos \theta \cdot \cos \psi-\sin \varphi \cdot \sin \psi & -\cos \varphi \cdot \sin \theta \\
\sin \theta \cdot \sin \psi & \sin \theta \cdot \cos \psi & \cos \theta
\end{array}\right|, \\
\Phi_{x y z} & =\left|\begin{array}{ccc}
\cos \varphi \cdot \cos \psi-\cos \theta \cdot \sin \varphi \cdot \sin \psi & \cos \varphi \cdot \cos \theta \cdot \sin \psi+\cos \psi \cdot \sin \varphi & \sin \theta \cdot \sin \psi \\
-\cos \varphi \cdot \sin \psi-\sin \varphi \cdot \cos \varphi \cdot \cos \psi & \cos \varphi \cdot \cos \theta \cdot \cos \psi-\sin \varphi \cdot \sin \psi & \sin \theta \cdot \cos \psi \\
\sin \varphi \cdot \sin \theta & -\cos \varphi \cdot \sin \theta & \cos \theta
\end{array}\right| .
\end{aligned}
$$

Therefore, the expression, $I=C\left|\mathbf{e}_{i} \cdot \bar{\Phi}_{x y z} \mathbf{R}_{j} \Phi_{x y z} \cdot \mathbf{e}_{s}\right|^{2}$, can be used to calculate the Raman intensity in the specimen coordinate system.

In the parallel polarization $z(y y) \bar{z}$ and cross polarization $z(y x) \bar{z}$ of backscattering geometry, the unit polarization vectors in specimen coordinate system can be expressed as

$$
\begin{aligned}
\mathbf{e}_{i} & =\left(\begin{array}{lll}
0 & 1 & 0
\end{array}\right), \\
\mathbf{e}_{s}^{\|} & =\left(\begin{array}{lll}
0 & 1 & 0
\end{array}\right)^{\mathrm{T}}, \\
\mathbf{e}_{s}^{\perp} & =\left(\begin{array}{lll}
1 & 0 & 0
\end{array}\right)^{\mathrm{T}},
\end{aligned}
$$

where the superscript symbols $\|$ and $\perp$ refer to parallel and cross polarization configurations, respectively. 
Substituting (3), (4), and (6) into (2), the Raman intensities at $A_{1}, E_{1}$, and $E_{2}$ bands are obtained as follows:

$$
\begin{aligned}
& I_{A}^{\|} \propto\left[a^{\prime}-\left(a^{\prime}-b^{\prime}\right) \sin ^{2} \theta \cdot \cos ^{2} \psi\right]^{2}, \\
& I_{A}^{\perp} \propto\left[-\frac{\left(a^{\prime}-b^{\prime}\right)}{2} \sin 2 \psi \cdot \sin ^{2} \theta\right]^{2}, \\
& I_{E_{1}(x)}^{\|} \propto\left[2 c^{\prime} \cdot \sin \theta \cdot \cos \psi(\cos \varphi \cdot \sin \psi+\sin \varphi \cdot \cos \theta \cdot \cos \psi)\right]^{2},
\end{aligned}
$$

$I_{E_{1}(x)}^{\perp} \propto\left[c^{\prime} \cdot \sin \theta(-\cos \varphi \cdot \cos 2 \psi+\sin \varphi \cdot \cos \theta \cdot \sin 2 \psi)\right]^{2}$,

$I_{E_{1}(y)}^{\|} \propto\left[2 c^{\prime} \cdot \sin \theta \cdot \cos \psi(\sin \varphi \cdot \sin \psi-\cos \varphi \cdot \cos \theta \cdot \cos \psi)\right]^{2}$,

$I_{E_{1}(y)}^{\perp} \propto\left[c^{\prime} \cdot \sin \theta(\sin \varphi \cdot \cos 2 \psi+\cos \varphi \cdot \cos \theta \cdot \sin 2 \psi)\right]^{2}$,

$$
\begin{array}{r}
I_{E_{2}(x)}^{\|} \propto\left[2 d^{\prime}(\cos \varphi \cdot \sin \psi+\cos \theta \cdot \cos \psi \cdot \sin \varphi)\right. \\
\cdot(\sin \varphi \cdot \sin \psi-\cos \varphi \cdot \cos \theta \cdot \cos \psi)]^{2},
\end{array}
$$

$$
\begin{array}{r}
I_{E_{2}(x)}^{\perp} \propto\left[-d^{\prime}\left(\frac{1}{2} \sin 2 \varphi \cdot \sin 2 \psi\left(1+\cos ^{2} \theta\right)\right.\right. \\
-\cos \theta \cdot \cos 2 \varphi \cdot \cos 2 \psi)]^{2},
\end{array}
$$

$$
\begin{aligned}
I_{E_{2}(y)}^{\|} \propto\left[\begin{array}{l}
d^{\prime} \\
(
\end{array}\right. & (\cos \varphi \cdot \sin \psi+\cos \varphi \cdot \cos \psi \cdot \sin \varphi)^{2} \\
& \left.\left.-(\sin \varphi \cdot \sin \psi-\cos \varphi \cdot \cos \theta \cdot \cos \psi)^{2}\right)\right]^{2},
\end{aligned}
$$

$$
\begin{aligned}
I_{E_{2}(y)}^{\perp} \propto\left[-d^{\prime}(\right. & \frac{1}{2} \cos 2 \varphi \cdot \sin 2 \psi\left(1+\cos ^{2} \theta\right) \\
& +\cos \theta \cdot \sin 2 \varphi \cdot \cos 2 \psi)]^{2} .
\end{aligned}
$$

The proportional coefficients in (7), (8), (9), (10), (11), (12), (13), (14), (15), and (16) are the same. The Raman intensity at $E$ bands $\left(E_{1}\right.$ and $\left.E_{2}\right)$ consists of two components, $I_{E(x)}$ and $I_{E(y)}$, and they can be described according to the following equation [18]:

$$
I_{E}^{\perp \|}=n \cdot I_{E(x)}^{\perp \|}+(1-n) \cdot I_{E(y)}^{\perp \|},
$$

where weight factor $n=0.5$ [19]. By (17), the Raman intensity at the $E$ bands can be simplified as follows:

$$
I_{E_{1}}^{\|} \propto 2 c^{\prime 2} \cdot \sin ^{2} \theta \cdot \cos ^{2} \psi\left(\sin ^{2} \psi+\cos ^{2} \theta \cdot \cos ^{2} \psi\right),
$$

$$
\begin{aligned}
& I_{E_{2}}^{\|} \propto \frac{1}{2} d^{\prime 2}\left(\sin ^{2} \theta \cdot \sin ^{2} \psi-\sin ^{2} \theta+1\right)^{2}, \\
& I_{E_{1}}^{\perp} \propto \frac{1}{2} c^{\prime 2} \cdot \sin ^{2} \theta\left(\cos ^{2} 2 \psi+\cos ^{2} \theta \cdot \sin ^{2} 2 \psi\right), \\
& I_{E_{2}}^{\perp} \propto-\frac{1}{2} d^{\prime 2}\left(\sin ^{4} \theta \cdot \sin ^{4} \psi-\sin ^{4} \theta \cdot \sin ^{2} \psi-\cos ^{2} \theta\right) .
\end{aligned}
$$

Equations (7), (8), (18), (19), (20), and (21) describe the relationship of Raman intensities with $\theta$ and $\psi$ in different polarization configurations. For the sample with known crystallographic orientation, such as A-plane $\mathrm{ZnO}$ sample, the Raman intensities of different in-plane rotation angles, $\psi$, under the same value of $\theta$ are measured; the curves of Raman intensity versus $\psi$ were fitted by (7), (8), (18), (19), (20), and (21); the Raman tensor elements $\left(a^{\prime}, b^{\prime}, c^{\prime}\right.$, and $\left.d^{\prime}\right)$ can be obtained. Further, for the sample with unknown crystallographic orientation, the Raman intensities of different in-plane rotation angles, $\psi$, under the same value of $\theta$ are also measured. According to (7), (8), (18), (19), (20), and (21) and combining the Raman tensor elements, the curves of Raman intensity versus $\psi$ were fitted. Thus, the unknown Euler angles $\theta_{c}$ and $\psi_{c}$ can be estimated.

For the measurement of the Euler angle $\varphi_{c}$, it is necessary to change the polarization direction of incident light for many times. The polarization direction of the incident light can be adjusted by a $\lambda / 2$ wave plate. While incident light passes through the $\lambda / 2$ wave plate, the mirrors and the notch filter will affect its polarization direction. Suppose the primary optical axis of the $\lambda / 2$ wave plate is initially parallel to the polarization direction of incident light. Then, the polarization vector of the incident beam depends on the $\lambda / 2$ wave plate's rotation angle $(\gamma)$ and can be written as $[14]$

$$
\mathbf{e}_{s}=\left(\begin{array}{lll}
\cos \alpha, & r \cdot \sin \alpha, & 0
\end{array}\right),
$$

where $\alpha$ is twice the $\lambda / 2$ wave plate's rotation angle $(\alpha=2 \gamma)$ and $r$ is the correction factor of incident light $(0 \leq r \leq 1)$.

The mirrors and the notch filter will also affect the polarization direction of the scattering light. The effective polarization vector $\mathbf{e}_{s}$ of the scattering beam can be written as [14]

$$
\mathbf{e}_{s}=(m \cdot \cos \beta, \quad \sin \beta, \quad 0)^{\mathrm{T}},
$$

where $\beta$ is describing the analyzer position and $m$ is the correction factor of the scattered light.

In any coordinate system, the Raman intensity can be expressed as [14]

$$
I(\alpha, \beta, \varphi, \theta, \psi)=C\left|\mathbf{e}_{i} \cdot \bar{\Phi}_{x y z} \mathbf{R}_{j} \Phi_{x y z} \cdot \mathbf{e}_{s}\right|^{2} .
$$


Substituting (2), (3), (7), (22), and (23) into (24), a concise matrix equation is finally obtained.

$$
I(\alpha, \beta, \varphi, \theta, \psi)=C \cdot\left(\begin{array}{c}
\cos ^{2} \alpha \\
r \cdot \cos \alpha \cdot \sin \alpha \\
r^{2} \cdot \sin ^{2} \alpha
\end{array}\right)^{\mathrm{T}} \cdot\left(\begin{array}{lll}
f_{11} & f_{12} & f_{13} \\
f_{12} & f_{22} & f_{23} \\
f_{13} & f_{23} & f_{33}
\end{array}\right) \cdot\left(\begin{array}{c}
m^{2} \cdot \cos ^{2} \beta \\
m \cdot \cos \beta \cdot \sin \beta \\
\sin ^{2} \beta
\end{array}\right)
$$

where $f_{i j}=f_{i j}(\varphi, \theta, \psi)$ are the functions of $\varphi, \theta$, and $\psi$ $(i, j=1,2,3)$. The functions $f_{i j}$ for $E_{2}$ band are as follows:

$$
\begin{aligned}
& \begin{aligned}
& f_{11}(\varphi, \theta, \psi)= 4 \sin ^{2} \theta \cdot \sin ^{2} \psi(\cos \psi \cdot \sin \varphi-\cos \varphi \cdot \cos \psi+\cos \varphi \cdot \cos \theta \cdot \sin \psi+\cos \theta \cdot \sin \theta \cdot \sin \psi)^{2}, \\
& f_{12}(\varphi, \theta, \psi)= 4 \sin ^{2} \theta \cdot \sin \psi(\cos \psi \cdot \sin \varphi-\cos \varphi \cdot \cos \psi+\cos \varphi \cdot \cos \theta \cdot \sin \psi+\cos \varphi \cdot \cos \theta \cdot \sin \psi)(\cos \varphi+\sin \varphi) \\
& \cdot(\cos 2 \psi+\cos \theta \cdot \sin 2 \psi), \\
& f_{13}(\varphi, \theta, \psi)=-\sin ^{2} \theta\left(-2 \cos ^{4} \psi+\cos 2 \psi+16 \sin \psi \cdot \cos ^{2} \varphi \cdot \cos \theta \cdot \cos ^{3} \psi-8 \sin \psi \cdot \cos ^{2} \varphi \cdot \cos \theta \cdot \cos \psi+4 \sin 2 \varphi \cdot \cos ^{2} \theta\right. \\
& \quad \cdot \cos ^{4} \psi-4 \sin 2 \varphi \cdot \cos ^{2} \theta \cdot \cos ^{2} \psi+4 \sin 2 \varphi \cdot \cos ^{4} \psi-4 \sin 2 \varphi \cdot \cos ^{2} \psi-4 \cos ^{2} \theta \cdot \cos ^{2} \psi-8 \sin \psi \\
&\left.\quad \cdot \cos \theta \cdot \cos ^{3} \psi+4 \sin \psi \cdot \cos \theta \cdot \cos \psi-2 \cos ^{4} \psi+4 \cos ^{2} \theta \cdot \cos ^{4} \psi+2 \cos ^{2} \psi+\sin 2 \varphi\right), \\
& f_{22}(\varphi, \theta, \psi)=2 \sin ^{2} \theta\left(32 \sin \psi \cdot \cos ^{2} \varphi \cdot \cos \theta \cdot \cos ^{3} \psi-16 \sin \psi \cdot \cos ^{2} \varphi \cdot \cos \theta \cdot \cos \psi+8 \sin 2 \varphi \cdot \cos ^{2} \theta \cdot \cos ^{4} \psi-8 \sin 2 \varphi \cdot \cos ^{2} \theta\right. \\
& \quad \cdot \cos ^{2} \psi+8 \sin 2 \varphi \cdot \cos ^{4} \psi-8 \sin 2 \varphi \cdot \cos ^{2} \psi+8 \cos ^{2} \theta \cdot \cos ^{4} \psi-8 \cos ^{2} \theta \cdot \cos ^{2} \psi-16 \sin \psi \cdot \cos \theta \\
&\left.\quad \cdot \cos ^{3} \psi+8 \sin \psi \cdot \cos \theta \cdot \cos \psi-8 \cos ^{4} \psi+8 \cos ^{2} \psi+\sin 2 \varphi-1\right), \\
& f_{23}(\varphi, \theta, \psi)=2 \sin ^{2} \theta \cdot \sin 2 \psi\left(2 \sin { }^{2} \theta \cdot \sin ^{2} \psi-\sin { }^{2} \varphi \cdot \sin ^{2} \psi-2 \sin ^{2} \theta+\sin ^{2} \varphi \cdot \sin ^{2} \psi+1\right), \\
& f_{33}(\varphi, \theta, \psi)=-4\left(\cos \varphi \cdot \sin \psi-\sin \varphi \cdot \sin ^{2} \psi+\cos \varphi \cdot \cos \theta \cdot \cos \psi+\cos \theta \cdot \cos \psi \cdot \sin ^{2} \varphi\right)^{2} \cdot \cos ^{2} \psi \cdot \sin ^{2} \theta_{c} .
\end{aligned}
\end{aligned}
$$

In order to simplify $f_{i j}$, the values of $\theta_{c}$ and $\psi_{c}$ are substituted into the expression $f_{i j}$. Thus, we can explicitly obtain a set of general expressions that depend on $\varphi$.

For the accessible analyzer position $\left(\beta=0^{\circ}\right),(25)$ can be simplified as follows:

$$
\begin{aligned}
I_{x}=I\left(\alpha, 0^{\circ}, \varphi, \theta, \psi\right)=C & \left(f_{11} \cdot \cos ^{2} \alpha+r \cdot f_{12} \cdot \cos \alpha \sin \alpha+r^{2}\right. \\
& \left.\cdot f_{13} \cdot \sin ^{2} \alpha\right) \cdot m^{2} .
\end{aligned}
$$

To fit the experimental data, we use the following fitting functions [14]:

$$
I_{x}=U_{1} \cdot \cos ^{2} \alpha+U_{2} \cdot \sin 2 \alpha+U_{3} \cdot \sin ^{2} \alpha,
$$

where $U_{1}, U_{2}$, and $U_{3}$ are adjustable coefficients.

By comparing the coefficient of (27) and (28), we obtain the equations below:

$$
\begin{array}{r}
r^{2} \cdot u_{1} \cdot f_{13}(\varphi)-f_{11}(\varphi)=0, \\
r \cdot u_{2} \cdot f_{12}(\varphi)-f_{11}(\varphi)=0,
\end{array}
$$

where $u_{1}=U_{1} / U_{3}$ and $u_{2}=U_{1} / U_{2}$.

Solving the unknown parameters $\varphi$ and $r$ in (29) using the Gauss-Newton method, the value $\varphi_{c}$ can be determined.

\section{Experimental}

The samples analyzed in this work were the A-plane and $\mathrm{M}$-plane $\mathrm{ZnO}$ grown by the hydrothermal method. Both crystals were cut into square-shaped plates with a size of $5 \times 5 \mathrm{~mm}^{2}$ and a thickness of $0.5 \mathrm{~mm}$. The measurement surfaces of samples were polished to an optical grade. The crystallographic orientation of the studied crystal faces was preliminarily determined by means of X-ray diffraction analysis.

The macro-Raman measurements were performed at room temperature using a confocal Raman spectrometer (Renishaw InVia Reflex) with the $532 \mathrm{~nm}$ emission line of a 


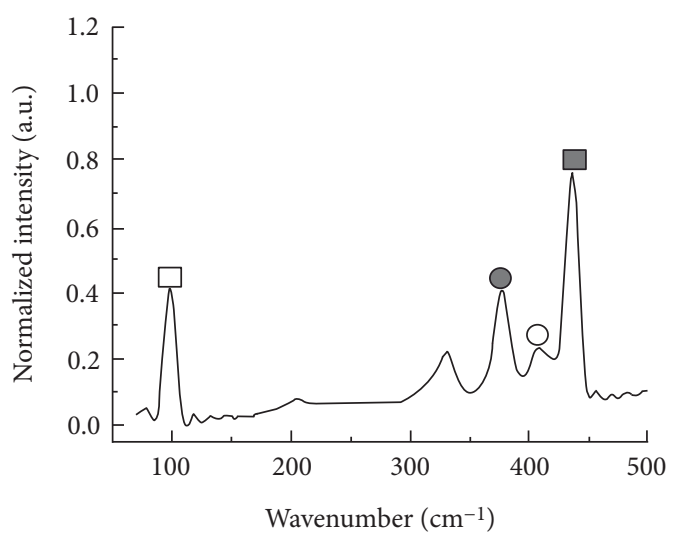

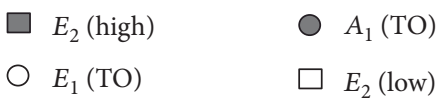

(a) A-plane

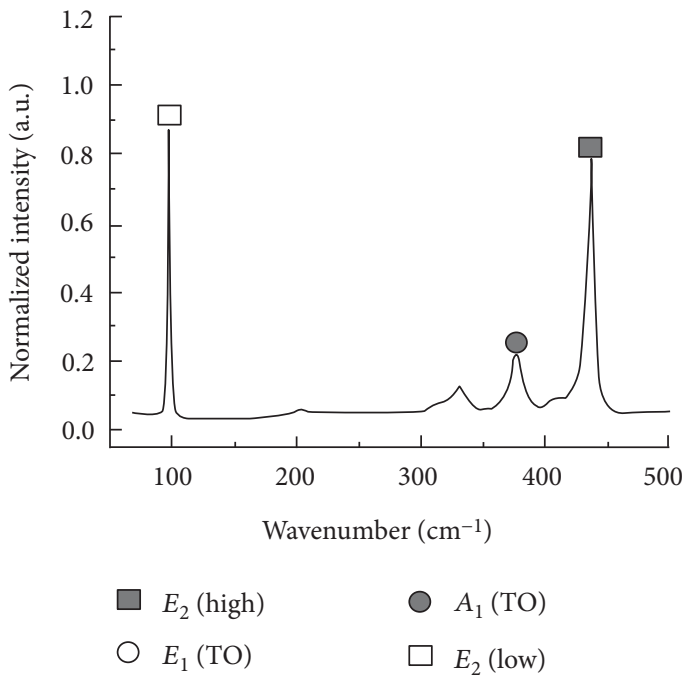

(c) A-plane

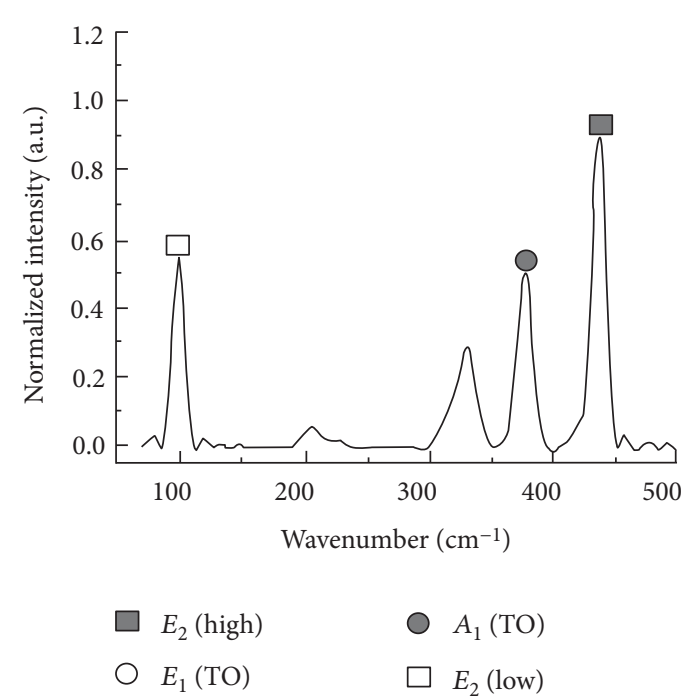

(b) M-plane

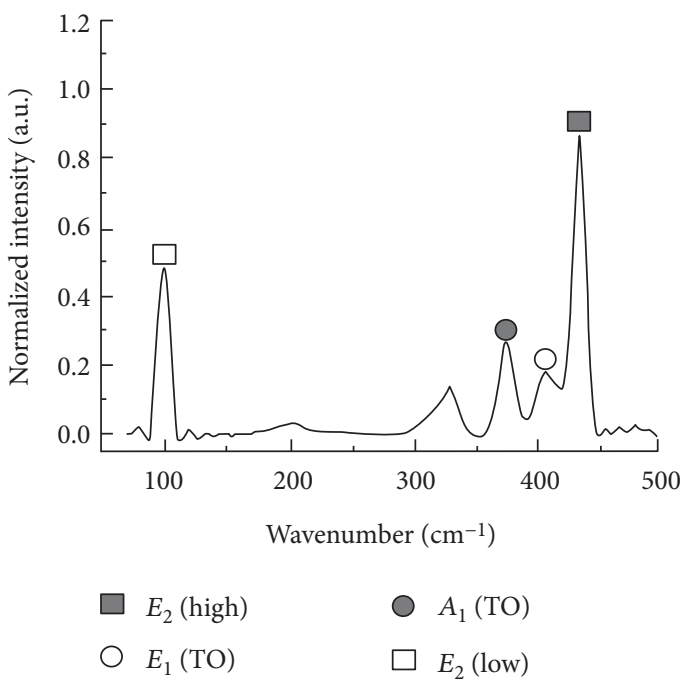

(d) M-plane

Figure 2: Polarized Raman spectra collected on A- and M-plane $\mathrm{ZnO}$ samples in parallel (a and b) and cross (c and d) polarization configurations.

semiconductor laser as excitation source. A $\times 100$ objective lens was adopted, and the spot diameter is less than $1 \mu \mathrm{m}$.

The $Y^{\mathrm{P}}$ axis (crystal orientation index was [0001]) of the specimen coordinate system of A-plane $\mathrm{ZnO}$ was horizontally placed on the experimental platform of Raman spectrometer, as shown in Figure 1(c). The A-plane sample was rotated counter-clockwise by 180 degrees by virtue of parallel polarization and cross polarization configuration, respectively. In the process of sample rotation, the Raman spectral data were collected every 10 degrees. The sample to be measured (M-plane) was placed on the Raman spectrometer in the same way as shown in Figure 1(c). The sample to be measured was rotated counterclockwise by 180 degrees through parallel polarization. In the process of sample rotation, the Raman spectral data were collected every 10 degrees. After that, the sample was rotated counterclockwise by 180 degrees, and 0 -degree polarization analyzer $(\beta=0$ degree) was used for the light path in Raman spectrometer. The $\lambda / 2$ wave plate was placed between the light source and the spectrometer box as shown in Figure 1(c). The $\lambda / 2$ wave plate was rotated clockwise by 90 degrees along the laser propagation direction. In the rotation process of the $\lambda / 2$ wave plate, Raman spectral data were collected every 5 degrees.

Raman spectra collected on the A-plane sample and M-plane sample of the wurtzitic $\mathrm{ZnO}$ single crystals with different polarized configurations are shown in Figure 2. We can clearly observe four first-order optical bands $\left(E_{2}\right.$ (low; $99 \mathrm{~cm}^{-1}$ ), $A_{1}$ (TO; $378 \mathrm{~cm}^{-1}$ ), $E_{1}$ (TO; $407 \mathrm{~cm}^{-1}$ ), and $E_{2}$ (high; $437 \mathrm{~cm}^{-1}$ )) of A-plane $\mathrm{ZnO}$ under the parallel polarization; $E_{2}$ (low; $\left.99 \mathrm{~cm}^{-1}\right), A_{1}\left(\mathrm{TO} ; 378 \mathrm{~cm}^{-1}\right)$, and $E_{2}$ (high; $437 \mathrm{~cm}^{-1}$ ) bands can be observed under the cross 


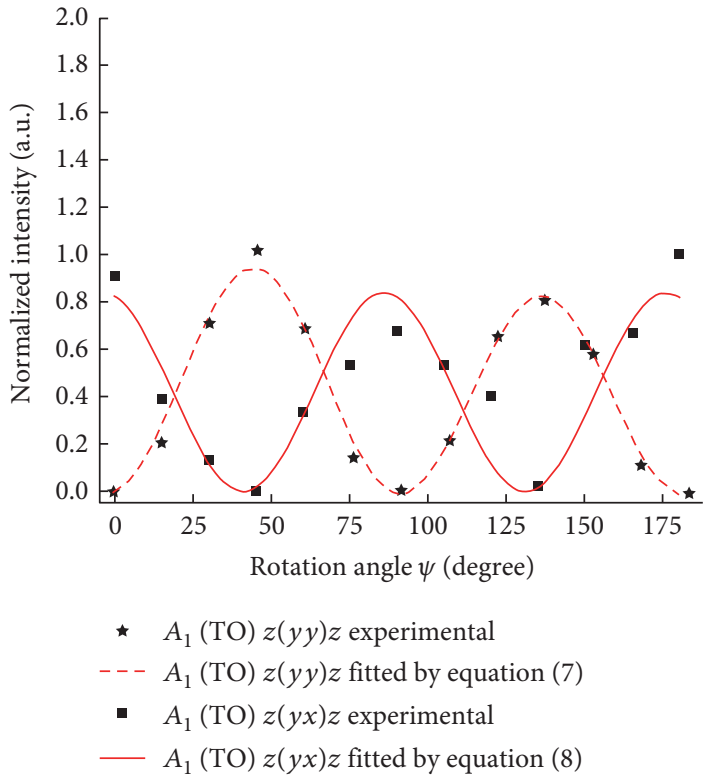

(a)

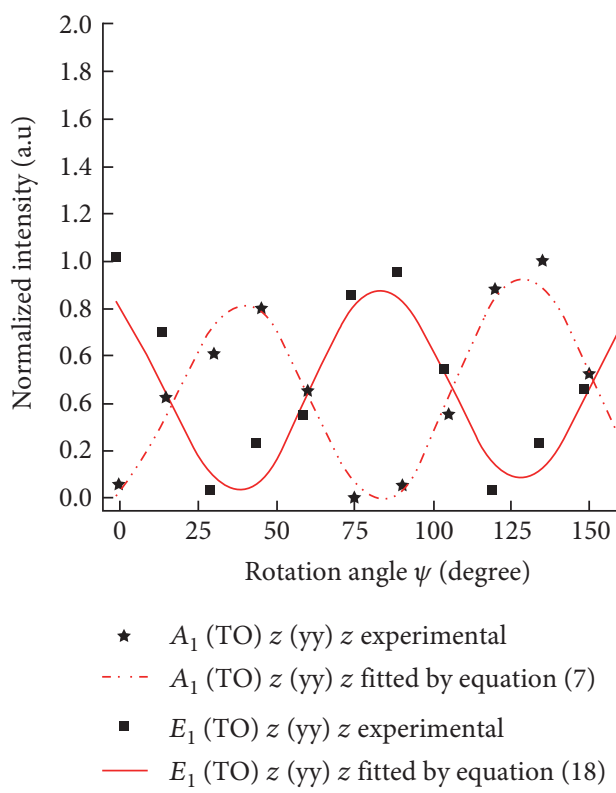

(c)

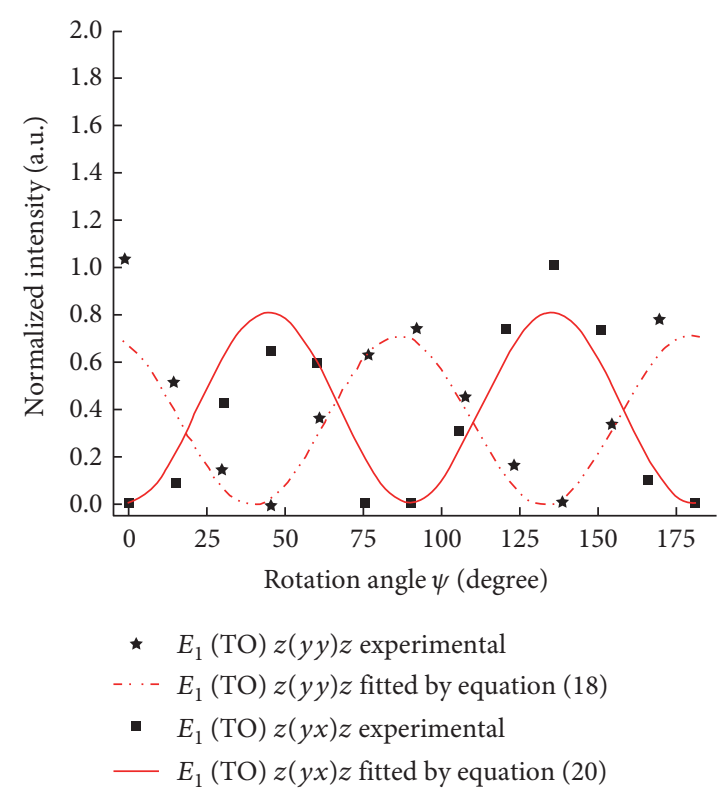

(b)

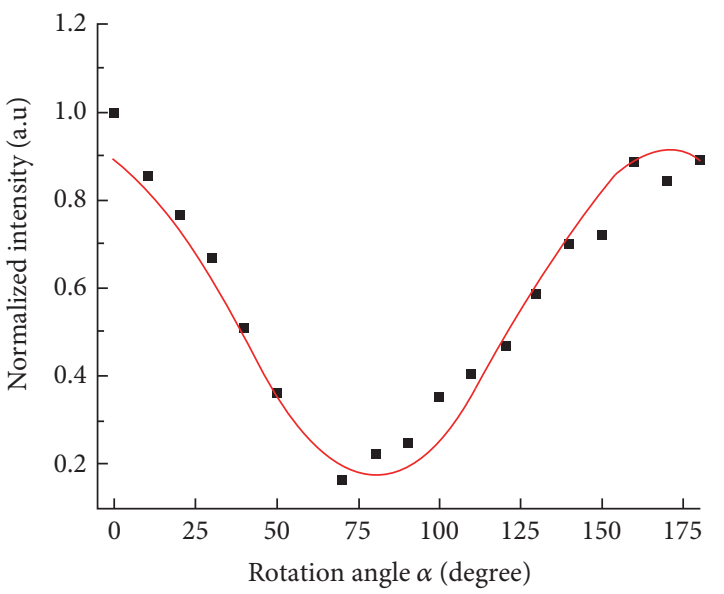

- Experimental

- Fitted by equation (29)

(d)

Figure 3: Change of Raman intensities at (a) $A_{1}$ (TO) and (b) $E_{1}$ (TO) bands of A-plane ZnO with the rotation angle $\psi$; (c) change of intensities at $A_{1}(\mathrm{TO})$ and $E_{1}$ (TO) bands of M-plane $\mathrm{ZnO}$ under parallel polarization with the rotation angle $\psi$; (d) change of intensities at $E_{2}$ (low) band of $\mathrm{M}$-plane $\mathrm{ZnO}$ with the polarization direction of incident light.

polarization. As to the $\mathrm{M}$-plane $\mathrm{ZnO}$, we can clearly observe three first-order optical bands, $E_{2}$ (low), $A_{1}$ (TO), and $E_{2}$ (high), under the parallel polarization and four first-order optical bands, $E_{2}$ (low), $A_{1}$ (TO), $E_{1}$ (TO), and $E_{2}$ (high), under the cross polarization. The observed vibration bands are in good agreement with the theoretical calculation results based on the polarization selection rules.

Figures 3(a) and 3(b) show the experimentally detected intensity variations at $A_{1}$ (TO) and $E_{1}$ (TO) bands of the A-plane $\mathrm{ZnO}$ single crystal $\left(\theta=90^{\circ}\right)$ as a function of in-plane rotation angle $\psi$. Raman intensities collected at the $A_{1}$ (TO) band under both parallel and cross polarization were fitted by theoretical equations (7) and (8), according to the least-square method. The constants $a^{\prime}=0.91$ and $b^{\prime}=-0.935$ were then obtained. An average value of the constant $c^{\prime}, c^{\prime}=1.275$, was extracted from the results of fitting the experimental data in Figure 3(b) by (18) and (20).

Figure 3(c) shows the intensity variations at $A_{1}(\mathrm{TO})$ and $E_{1}$ (TO) bands of M-plane $\mathrm{ZnO}$ single crystal as a function of the in-plane rotation angle $\psi$. Substituting the values of $a^{\prime}, b^{\prime}$, 
and $c^{\prime}$ into (7) and (18), the angular dependence of the intensity of the Raman bands was fitted according to the least-square method and then we got the values of $\psi_{c}$ and $\theta_{c}\left(\psi_{c}=128.97^{\circ}, \theta_{c}=89.87^{\circ}\right.$ and $\left.\psi_{c}=128.97^{\circ}, \theta_{c}=97.0^{\circ}\right)$. Their average values are $\psi_{c}=128.93^{\circ}$ and $\theta_{c}=93.44^{\circ}$, respectively. Raman intensity at the $E_{2}$ (low) band of M-plane $\mathrm{ZnO}$ changed with the polarization direction of incident light, $\alpha$, as shown in Figure $3(\mathrm{~d})$. The values of $U_{1}=0.89$, $U_{2}=-0.126$, and $U_{3}=0.194$ were obtained from a leastsquare fitting of the plot shown in Figure 3(d). Substituting the values of $u_{1}$ and $u_{2}\left(u_{1}=4.75\right.$ and $\left.u_{2}=-7.06\right)$ into the equation system (29), the value of the Euler angle $\varphi_{c}$ can be obtained $\left(\varphi_{c}=-3.67^{\circ}\right)$

The Miller index of M-plane sample used in the experiment is $(10 \overline{1} 0)[1 \overline{1} 00]$. The theoretical values of Euler angles calculated by (1) are $\varphi_{c}=0^{\circ}, \theta_{c}=90^{\circ}$, and $\psi_{c}=120^{\circ}$. These values are in agreement with experimental values $\left(\varphi_{c}=-3.67^{\circ}, \theta_{c}=93.44^{\circ}\right.$, and $\left.\psi_{c}=128.93^{\circ}\right)$. Therefore, the measurement of Euler angles of wurtzitic $\mathrm{ZnO}$ by Raman spectroscopy is feasible.

\section{Conclusions}

Based on the polarization selection rule and coordinate transformation theory, the step-by-step method for the Euler angle of the crystal of wurtzitic $\mathrm{ZnO}$ is proposed. A series of theoretical formulas regarding the Euler angle measurement were deduced. The specific experimental processes were given. The measurement of the Raman tensor elements and Euler angles of a $\mathrm{ZnO}$ crystal were achieved. The results show a trigonometric functional relationship between the intensities at each Raman band of wurtzite crystal and the Euler angle, $\psi$, under different polarization configurations. Fitting the curves of measured Raman intensity versus $\psi$, the Raman tensor elements and Euler angles $\theta$ and $\psi$ can be obtained. There is also a trigonometric functional relationship between the intensities at each Raman band and the polarization direction of incident light, which can be used to measure the Euler angle $\varphi$. The experimental results show that the method proposed in this paper can effectively realize the measurement of Euler angles for wurtzite crystal. Our investigation provides the approach for the nondestructive measurement of crystal orientation on the microscale, which can be used to determine the crystallographic orientation of small crystals, local micro regions of crystals, and thin epitaxial films.

\section{Conflicts of Interest}

The authors declare that there is no conflict of interests regarding the publication of this paper.

\section{Acknowledgments}

The authors acknowledge the financial support from the National Natural Science Foundation of China (nos. 11302149 and 11422219), the Tianjin Application Foundation and Frontier Technology Research Program (no. 15JCQNJC05000), the Natural Science Foundation of Hebei Province (no. A2016202195), and the Innovation Team
Training Plan of Tianjin Universities and Colleges (no. TD12-5043).

\section{References}

[1] P. Zu, Z. K. Tang, G. K. L. Wong et al., "Ultraviolet spontaneous and stimulated emissions from $\mathrm{ZnO}$ mic-rocrystallite thin films at room temperature," Solid State Communications, vol. 108, no. 8, pp. 459-463, 1997.

[2] M. H. Huang, S. Mao, H. Feick et al., "Room-temperature ultraviolet nanowire nanolasers," Science, vol. 292, no. 5523, pp. 1897-1899, 2001.

[3] M. Q. Wang, Y. Q. Lian, and X. G. Wang, "PPV/PVA/ZnO nanocomposite prepared by complex precursor method and its photovoltaic application," Current Applied Physics, vol. 9, no. 1, pp. 189-194, 2009.

[4] G. E. Bacon, N. A. Curry, and S. A. Wilson, "A crystallographic study of solid benzene by neutron diffraction," Proceedings of the Royal Society of London A: Mathematical and Physical Sciences, vol. 279, no. 1376, pp. 98-110, 1964.

[5] M. Zucali, M. Voltolini, B. Ouladdiaf, L. Mancini, and D. Chateigner, "The 3D quantitative lattice and shape preferred orientation of amylonitised metagranite from Monte Rosa (Western Alps): combining neutron diffraction texture analysis and synchrotron X-ray microtomography," Journal of Structural Geology, vol. 63, no. 3, pp. 91-105, 2014.

[6] M. Calcagnotto, D. Ponge, E. Demir, and D. Raabe, "Orientation gradients and geometrically necessary dislocations in ultrafine grained dual-phase steels studied by $2 \mathrm{D}$ and $3 \mathrm{D}$ EBSD," Materials Science and Engineering A, vol. 527, no. 10-11, pp. 2738-2746, 2010.

[7] S. L. Shrestha, A. J. Breen, P. Trimby, G. Proust, S. P. Ringer, and J. M. Cairney, "An automated method of quantifying ferrite microstructures using electron backscatter diffraction (EBSD) data," Ultramicroscopy, vol. 137, no. 1, pp. 40-47, 2014.

[8] A. Eghlimi, M. Shamanian, M. Eskandarian, A. Zabolian, M. Nezakat, and J. A. Szpunar, "Evaluation of microstructure and texture across the welded interface of super duplex stainless steel and high strength low alloy steel," Surface \& Coatings Technology, vol. 264, pp. 150-162, 2015.

[9] K. Gao, S. Li, L. Xu, and H. Fu, "Effect of solidification rate on microstructures and orientations of $\mathrm{Al}-\mathrm{Cu}$ hypereutectic alloy in thin crucible," Crystal Research \& Technology, vol. 49, no. 2-3, pp. 164-170, 2014.

[10] F. Li, L. Jin, Z. Xu, and Z. Guo, "Determination of threedimensional orientations of ferroelectric single crystals by an improved rotating orientation x-ray diffraction method," Review of Scientific Instruments, vol. 80, no. 8, pp. 085106-1085106-5, 2009.

[11] K. Mizoguchi and S. Nakashima, "Determination of crystallographic orientations in silicon films by Raman microprobe polarization measurements," Journal of Applied Physics, vol. 65 , no. 7, pp. 2583-2590, 1989.

[12] J. B. Hopkins and L. A. Farrow, "Raman microprobe determination of local crystal orientation," Journal of Applied Physics, vol. 59, no. 1103, pp. 1103-1110, 1986.

[13] M. C. Munisso, W. L. Zhu, and G. Pezzotti, "Raman tensor analysis of sapphire single crystal and its application to define crystallographic orientation in polycrystalline alumina," Physica Status Solidi B-Basic Solid State Physcics, vol. 246, no. 8, pp. 893-1900, 2009. 
[14] M. Becker, H. Scheel, and S. Christiansen, "Grain orientation, texture, and internal stress optically evaluated by microRaman spectroscopy," Journal of Applied Physics, vol. 101, no. 063531, pp. 1-10, 2007.

[15] W. R. Busing and H. A. Levy, "Angle calculations for 3- and 4circle X-ray and neutron diffractometers," Acta Crystallographica, vol. 22, no. 4, pp. 457-464, 1967.

[16] T. D. Tell and S. Porto, "Raman effect in cadmium sulfide," Physical Review, vol. 144, no. 2, pp. 771-774, 1966.

[17] R. Loudon, "The Raman effect in crystals," Advances in Physics, vol. 13, no. 52, pp. 423-482, 1964.

[18] G. Pezzotti, H. Sueoka, and A. A. Porporati, "Raman tensor elements for wurtzitic GaN and their application to assess crystallographic orientation at film/substrate interfaces," Journal of Applied Physics, vol. 110, no. 013527, pp. 1-10, 2009.

[19] K. Nakamoto, Infrared and Raman Spectra of Inorganic and Coordination Compounds, pp. 56-98, Wiley, New York, 1986. 

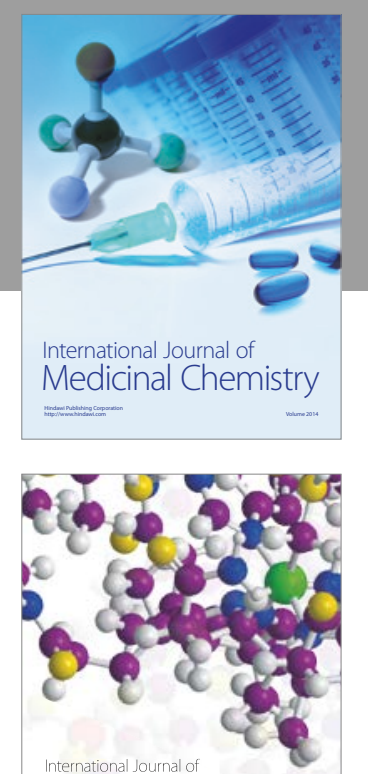

Carbohydrate Chemistry

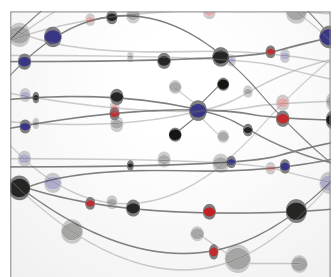

The Scientific World Journal
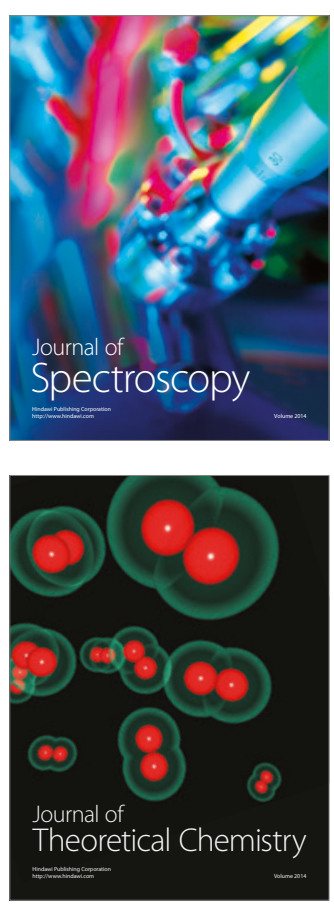
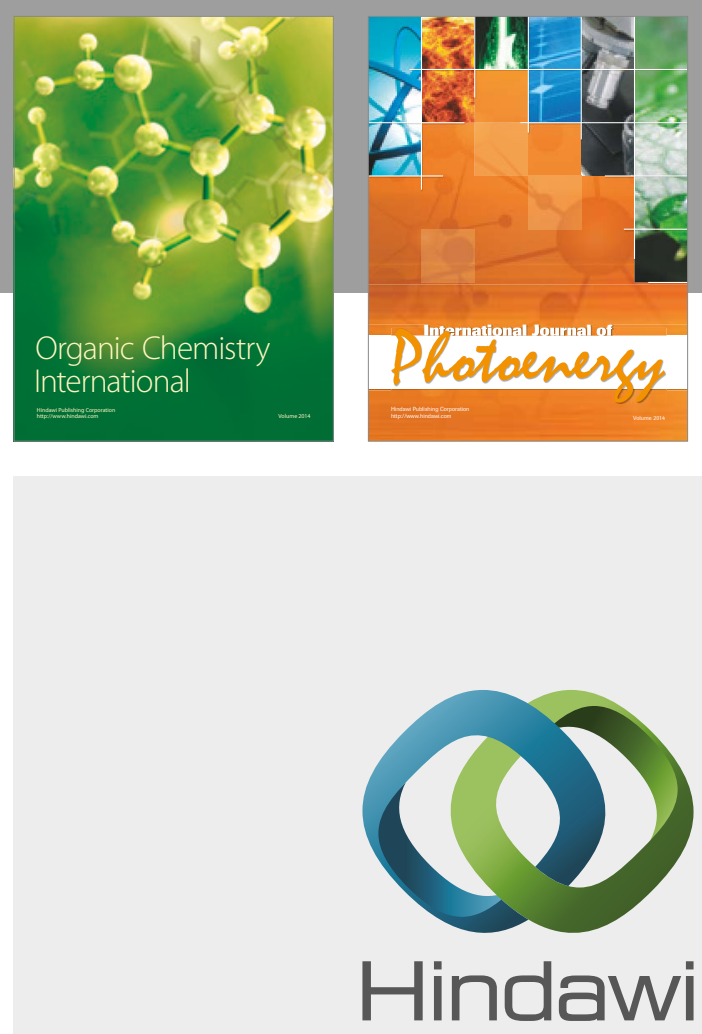

Submit your manuscripts at

https://www.hindawi.com

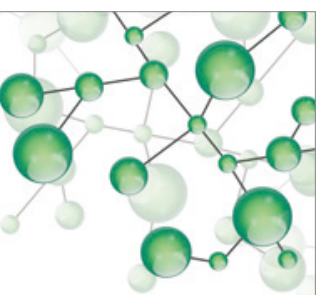

International Journal of

Inorganic Chemistry

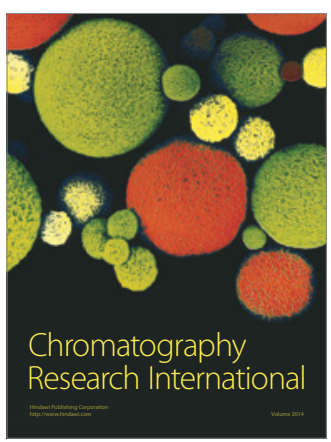

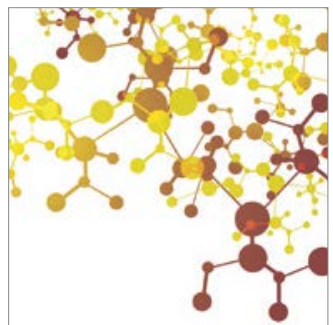

Applied Chemistry
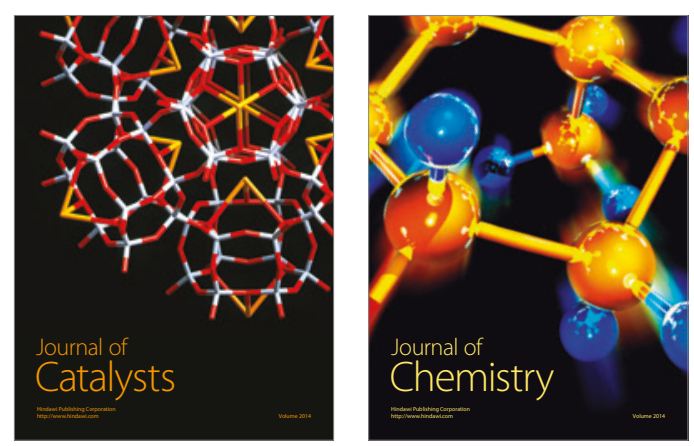
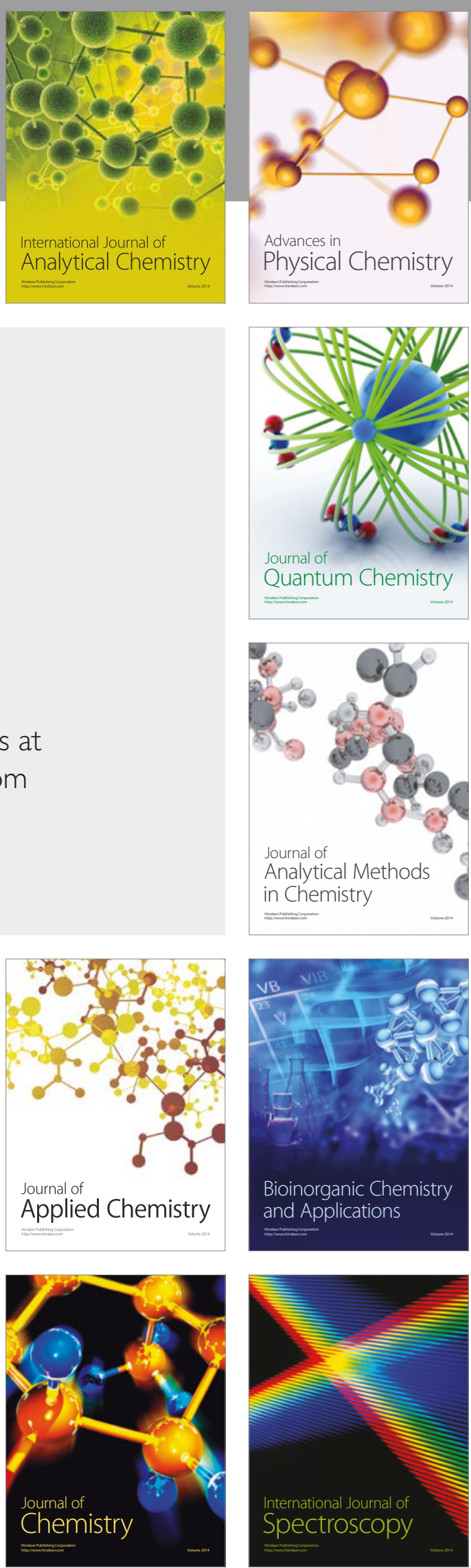